\title{
Effects of the interstrip gap on the efficiency and response of Double Sided Silicon Strip Detectors
}

\author{
D. Torresi ${ }^{1}$, J. Forneris ${ }^{2}$, L. Grassi $^{3}$, L. Acosta $^{1}$, A. Di Pietro ${ }^{1}$, \\ P. Figuera ${ }^{1}$, M. Fisichella ${ }^{1}$, V. Grilj ${ }^{2}$, M. Jakic ${ }^{3}$, \\ M. Lattudada ${ }^{1,4}$, T. Mijatovic ${ }^{3}$, M. Milin $^{5}$, L. Prepolec ${ }^{3}$,

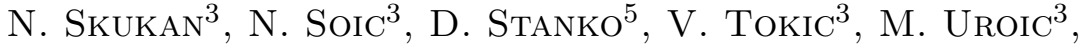 \\ M. ZADRO ${ }^{3}$
}

${ }^{1}$ INFN - Laboratori Nazionali del Sud, Catania, Italy

${ }^{2}$ Ruder Bošković Institute, Zagreb, Croatia

${ }^{3}$ Physics Department and NIS Interdepartmental Centre, University of Torino; INFN, Sezione di Torino, CNISM, Sezione di Torino, Italy

${ }^{4}$ Dipartimento di Fisica e Astronomia, Università di Catania, Catania, Italy

${ }^{5}$ Department of Physics, University of Zagreb, Zagreb, Croatia.

\begin{abstract}
In this work the effects of the segmentation of the electrodes of Double Sided Silicon Strip Detectors (DSSSDs) are investigated. In order to characterize the response of the DSSSDs we perform a first experiment by using tandem beams of different energies directly sent on the detector and a second experiment by mean of a proton microbeam. Results show that the effective width of the inter-strip region and the efficiency for full energy detection, varies with both detected energy and bias voltage. The experimental results are qualitatively reproduced by a simplified model based on the Shockley-Ramo-Gunn framework.
\end{abstract}




\section{Introduction}

Highly segmented double sided silicon detectors (DSSSD) are widely used in nuclear physics to perform accurate measurements of angular distributions, or to study reactions where coincidences of different particles are required to fully characterize the final state of the interaction process. The DSSSD under study in this work is the model W1, manufactured by Micron Semiconductor Ltd. It has an active area of $50 \times 50 \mathrm{~mm}^{2}$, each electrode is divided into 16 strips, vertical for the front side and horizontal for the back side. Each strip is $3 \mathrm{~mm}$ wide and they are separated by a $\mathrm{SiO}_{2}$ layer $1 \mu \mathrm{m}$ wide that in the following is called interstrip. it is known that that particles entering the detector through the $\mathrm{SiO}_{2}$ insulating interstrip region, can produce signals with an amplitude which is different than the full energy and also signals with an opposite polarity [1-6]. For this reason it is very important to be able to reject interstrip events by selecting only the events producing the correct full energy signals. Therefore, with the aim to characterize the DSSSD we performed [1] a systematic study to investigate the efffects of the different incident ion, energy, and polarization voltage on the efficiency for the full energy detection. Moreover we tried to identify an appropriate selection procedure of events which allows to maximize the efficiency for the full energy detection.

\section{Measurements of full energy detection efficiency}

A first experiment was performed at the Laboratori Nazionali del Sud, Catania, with the aim to study the dependence of the interstrip effects on the beam energy, the ion species and polarization voltages. ${ }^{7} \mathrm{Li}$ and ${ }^{16} \mathrm{O}$ Tandem beams at energies between 10 and $50 \mathrm{MeV}$, with intensity of about 100 pps, were directly sent onto a $1000 \mu \mathrm{m}$ thick DSSSD placed along the beam direction.

From the energy spectra collected is observed that some particle is detected with an energy lower than the full one. In fact particles whose trajectory crosses an interstrip region give rise to signals in the two adjacent strip and has a lower amplitude and sometimes opposite polarity $[5,6]$. In particular for particles crossing a back interstrip region (back inter-strip events) summing the signals from the two adjacent strips we obtain a full energy signal. Also for front inter-strip events two adjacent front strips give signals, but in this case one of them can have an opposite polarity and the sum of the two signals does not give a full energy signal. 


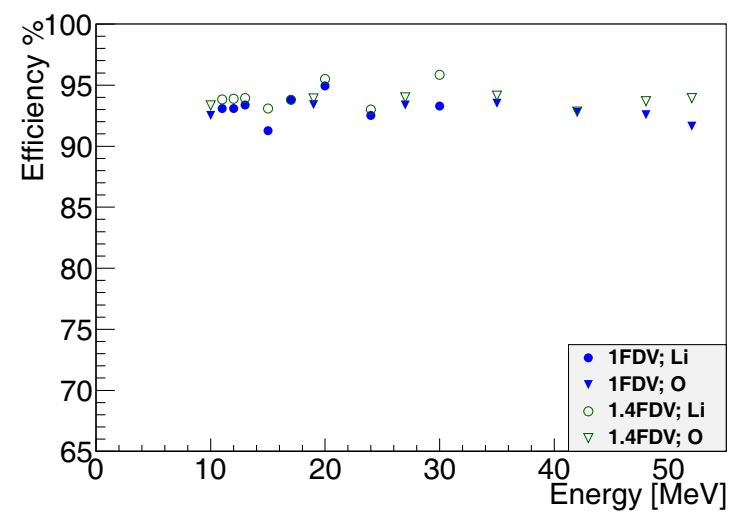

Figure 1: Efficiency for full energy detection as a function of the beam energy, for two different bias voltages applied to the detector. In the left panel is shown the efficiency obtained imposing the condition $\mathrm{E}_{\text {front }} \approx \mathrm{E}_{\text {back }}$, the continuous line corresponds to the efficiency for the full energy detection calculated using the geometrical interstrip width; in the right panel is shown the efficiency obtained recovering the back interstrip events. See the text for more details.

Therefore for data gathered with DSSSD, it is necessary a procedure to select the correct full energy events rejecting the others. A possible procedure for selecting the full energy events is to compare the energy measured by the front side $\mathrm{E}_{\text {front }}$ with the one measured by the back side $\mathrm{E}_{\text {back }}$ by imposing $\mathrm{E}_{\text {front }} \approx \mathrm{E}_{b a c k}$, within a given tolerance. Using this selection procedure we obtained an efficiency that is slightly dependent on the energy and on the bias applied on the detector as shown in figure 1 We proposed another events selection where the energy measured by the front strips is compared to the sum of the energy of two adjacent back strips. In this way all the back inter-strip events are recovered increasing the efficiency and removing the dependence on energy and on bias as shown in right panel of figure 1 [1]. The average value of the efficiency for full energy detection is $\sim 94 \%$.

\section{Measurements of the effective inter-strip width}

The efficiency for full energy detection is directly related with the effective inter-strip width, therefore we performed a second experiment with the aim to have a direct measurements of the effective interstrip width for different operating conditions of the DSSSD. The experiment was performed at the 
Ruder Bošković Institute in Zagreb using the local microbeam facility. The use of a microbeam allows to know the position of the impinging particle with a precision of few $\mu \mathrm{m}$, in this way it is possible to correlate the presence of interstrip effects with the impinging position of the particle on the DSSSD. Proton microbeams of 1.7 and $6 \mathrm{MeV}$ at low intensity ( $\sim 100 \mathrm{pps}$ ) were used to irradiate the DSSSD. For each beam energy the measurements were repeated for bias voltages equal to $0.5,1$ and 1.5 times the full depletion voltage (FDV).

In the left panel of figure 2 is shown the measured energy as a function of
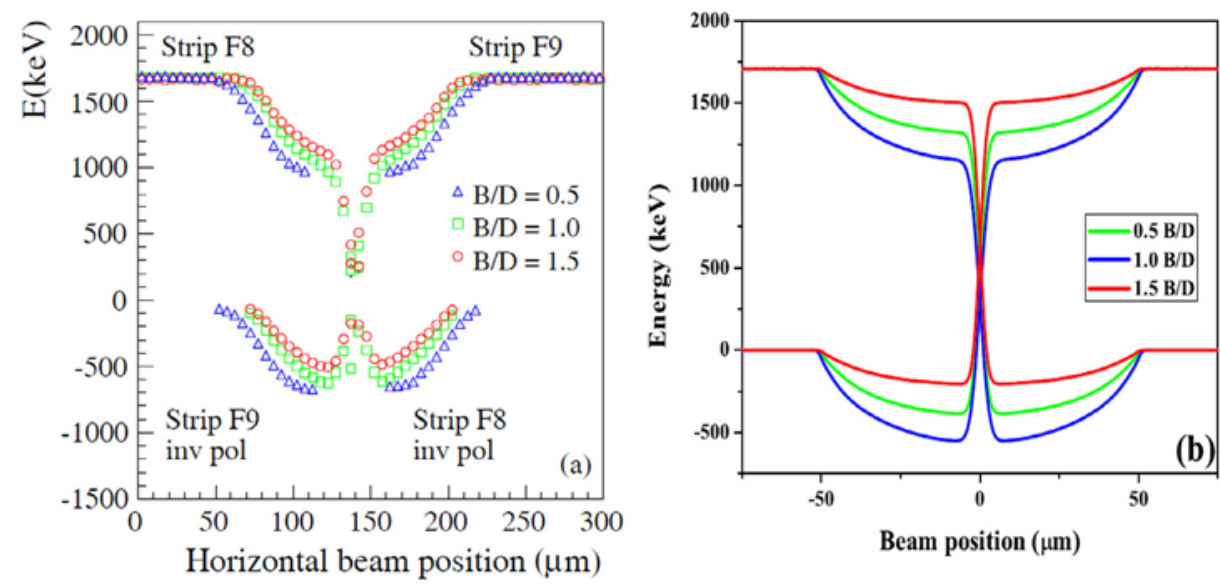

Figure 2: Energy as function of position for proton of $1.7 \mathrm{MeV}$ for three different bias voltages. On the left panel are shown experimental data refering to a front interstrip region, on the right panel are shown data coming from simulations (fro more information see the text). B/D indicates the ratio between the applied bias and the full depletion voltage.

the impinging position of the incident proton. From the plot it is possible to distinguish clearly the interstrip regions corresponding to the region where the amplitude of the energy signal drops. In order to provide a qualitative interpretation of the experimental findings a simplified simulation based on the Shockley-Ramo-Gunn framework was performed. As suggested in [6] it was assumed a build up of positive charge at the oxide interface in the front inter-strip and of negative charge at the oxide interface in the back inter-strip. In the right side of figure 2 we show the result of the simulation, a satisfactory qualitative reproduction of all the observed inter-strip effects has been obtained [2]. 


\section{Summary and conclusions}

The response of Double Sided Silicon Strip Detectors and its dependence on the incident ion type, energy, and polarization voltage was studied in two different experiments. A first study was performed by mean of ${ }^{7} \mathrm{Li}$ and ${ }^{16} \mathrm{O}$ tandem beams at different energies. It showed that the efficiency for full energy detection depends on the energy and type of the detected ion and on the applied bias voltage. A selection procedure of events that maximize the efficiency for the full energy reconstruction and remove the cited dependences has been proposed. In a second study, a proton micro-beams was used to perform a direct measurements of the effective interstrip width at different energies and for different detector bias. The results confirmed that both front and back effective inter-strip widths, which are directly related to the efficency for full energy detection, can be much larger than the nominal geometric width of the $\mathrm{SiO}_{2}$ zone and depend on the operating conditions. Finally the experimental observations were qualitatively interpreted and described by performing a simplified simulations based on the Shockley-Ramo-Gunn framework. Concluding, for those experiments aiming at an accurate measurements of absolute cross-sections, especially if two or more DSSSD are used in coincidence, efficiency for full energy detection cannot be deduced from the geometric inter-strip width but a characterization of the used DSSSDs is required.

\section{Acknowledgements}

This work has been partly supported by the European Community as an Integrating Activity "Support of Public and Industrial Research Using Ion Beam Technology - SPIRIT" (EC Contract no. 227012) by the European Community FP7 REGPOT project Particle Detectors (EC Contract no. 256783) and by the Italian Ministry of Education and Research (PRIN 20102011, project 2010TP-SCSP).

\section{References}

[1] Torresi et al., Nucl. Instr. Meth. A 713 (2013) 11.

[2] Grassi et al. Nucl. Instr. Meth. A 767 (2014) 99.

[3] Poehlsen, et al., Nucl. Instr. Meth.A 721 (2013) 26. 
[4] Takeda et al., Nucl. Instrum. Meth. Phys. Res. A579 859, (2007).

[5] Blumenfeld et al., Nucl. Instrum. Meth. Phys. Res. A421, 471 (1999).

[6] Yorkstone et al., Nucl. Instrum. Meth. Phys. Res. A262, 353 (1987). 